Supporting Information for

\title{
Formation of Nanoscale Composites of Compound Semiconductors Driven by Charge Transfer
}

Weiwei Gao, ${ }^{1}$ Roberto dos Reis,,${ }^{1,2}$ Laura T. Schelhas, ${ }^{3}$ Vanessa L. Pool, ${ }^{3}$ Michael F. Toney, ${ }^{3}$ Kin Man $\mathrm{Yu},{ }^{1,4}$ and Wladek Walukiewicz ${ }^{1 *}$

${ }^{1}$ Materials Sciences Division, Lawrence Berkeley National Laboratory, Berkeley, California 94720, USA

${ }^{2}$ National Center for Electron Microscopy, Molecular Foundry, Lawrence Berkeley National Laboratory, Berkeley, California 94720, USA

${ }^{3}$ Stanford Synchrotron Radiation Lightsource, SLAC National Accelerator Laboratory, Menlo Park, California 94025, USA

${ }^{4}$ Department of Physics and Materials Science, City University of Hong Kong, Kowloon, Hong Kong

*To whom correspondence should be addressed.

E-mail: w_walukiewicz@lbl.gov 


\section{Electronic band structure and band offsets between $\mathrm{CdO}$ and SnTe.}

a

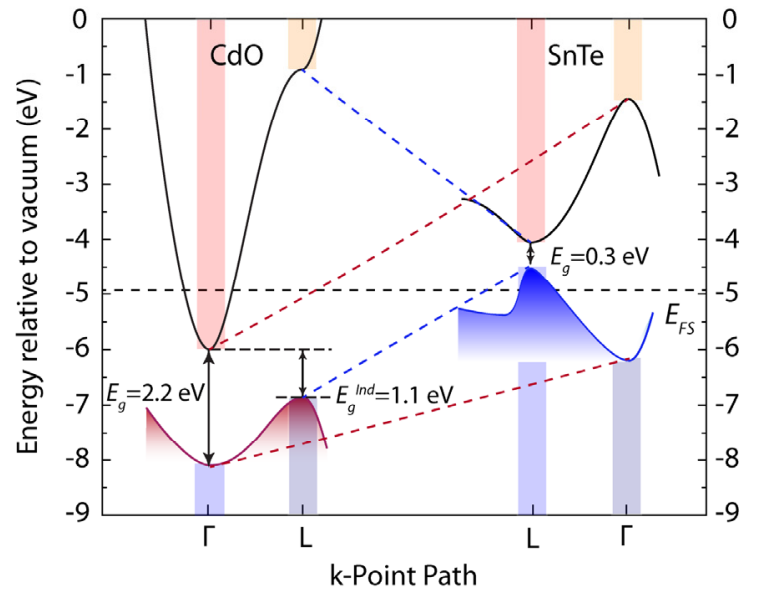

b

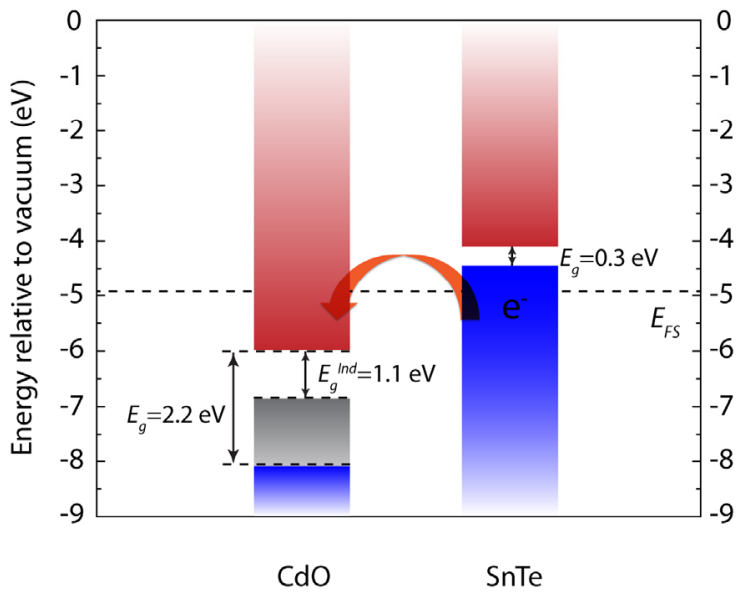

Figure S1. Band structure and band offsets between $\mathrm{CdO}$ and SnTe. (a) Calculated band structures of $\mathrm{CdO}^{1}$ and $\mathrm{SnTe}^{2}$ placed on the absolute energy scale using the Fermi level stabilization energy, $E_{F S}$ (black dashed line) as a common energy reference. Optical gaps $E_{g}$ of CdO and SnTe are highlighted. The red and blue dashed lines show the linear interpolations for conduction band edge $(\mathrm{CBE})$ and valence band edge (VBE) energies at $\Gamma$ and $\mathrm{L}$ point of the Brillouin zone. (b) Band offset and charge transfer at the $\mathrm{CdO} / \mathrm{SnTe}$ interface. The $\mathrm{CBE}$ of $\mathrm{CdO}$ at $5.9 \mathrm{eV}$ below the vacuum level and VBE of SnTe at $4.4 \mathrm{eV}$ below the vacuum level create an extreme type III band offset resulting in large electron transfer from the VBE of SnTe to the CBE of CdO.

Cadmium oxide $(\mathrm{CdO})$ is an example of the groups II -IV compound with extremely low conduction band edge (CBE) at $5.9 \mathrm{eV}$ below the vacuum level or $1 \mathrm{eV}$ below the $E_{F S}{ }^{3}$ Hence this material has a large propensity to n-type conductivity. The rock salt crystallographic structure $\mathrm{CdO}$ has a direct gap of $2.2 \mathrm{eV}$ in the $\Gamma$ point. The indirect band gap valence band maximum at the $\mathrm{L}$ point is $1.1 \mathrm{eV}$ below the $\Gamma$ point $\mathrm{CBE} .^{1}$

The valence band in SnTe presents a 4-fold degenerate valence band maximum lying at the L-point of the Brillouin zone and a lower energy 12-fold degenerate maximum at the $\sum$-point. ${ }^{4,5}$ 
The valence band edge (VBE) of SnTe at $\mathrm{L}$ point is located at $4.4 \mathrm{eV}$ below the vacuum level or $0.5 \mathrm{eV}$ above the $E_{F S}$ explaining the strong propensity of this material for p-type conductivity.

As shown in Fig. S1 the $\mathrm{CdO}$ and SnTe have an extreme type III band offset where the VBE of SnTe at L point is located at about $1.5 \mathrm{eV}$ above the $\mathrm{CBE}$ of $\mathrm{CdO}$. This leads to schematically shown in Fig. S1b large electron transfer from $\mathrm{SnTe}$ to $\mathrm{CdO}$ at the interface of the $\mathrm{CdO} / \mathrm{SnTe}$ heterostructure. There are no depletion regions or barriers for charge transfer at the $\mathrm{CdO} / \mathrm{SnTe}$ interface.

\section{Determination of the intrinsic band gap}

In the samples with large free charge carrier (electron or hole) concentration, the absorption edge energy is affected by the band filling effect known as the Burstein-Moss (BM) shift. ${ }^{6,7}$ In addition, as has been discussed previously ${ }^{6-9}$ the absorption edge energy is also affected by the interaction of free carriers as well as the interaction of charge carriers with the background of ionized centers.

For the n-type CdO-rich region, the relationship between electron concentration $n$ and the Fermi level energy is given by

$n=\frac{1}{3 \pi^{2}} \int_{0}^{\infty} \frac{\exp \left(z-\frac{E_{F}}{k_{B} T}\right)}{\left[1+\exp \left(z-\frac{E_{F}}{k_{B} T}\right)\right]^{2}} k^{3}(z) d z$,

Where $z=E_{C} / k_{B} T . k_{B}$ is the Bolzmann constant. $E_{c}$ is the electron energy with respect to the conduction band edge. $E_{F}$ is the Fermi level energy. The wave vector $k$ is determined by a nonparabolic dispersion relation, derived from Kane's two band $\boldsymbol{k} \cdot \boldsymbol{p}$ model ${ }^{10}$ 
$E(k)=\frac{\hbar^{2} k^{2}}{2 m_{0}}-\frac{E_{g}}{2}+\left(\sqrt{\left(\frac{E_{g}}{2}\right)^{2}+\frac{E_{g} \hbar^{2} k^{2}}{2 m_{e}^{*}}}\right)$,

Where $E_{g}$ is the intrinsic bandgap and $m_{e}{ }^{*}$ is the band edge effective mass. Here we assume the band edge effective mass of $m_{e}{ }^{*}=0.21 \mathrm{~m}_{0}$ for CdO-rich material. ${ }^{11}$ The carrier-induced renormalization effects due to the electron-electron correlation and electron-ion interactions can be determined by expressions in Refs. 7 and $8 .^{7,8}$

For p-type SnTe-rich region, the holes can occupy a 4-fold degenerate VBE lying at the L-

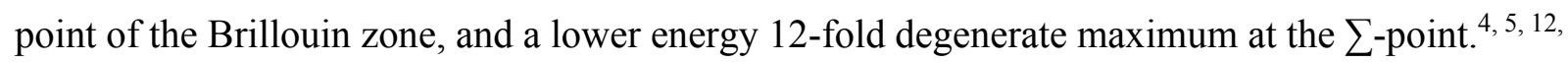
13

Therefore the relation between the holes concentration in the valence band and Fermi level energy given by

$p_{i}=\frac{1}{3 \pi^{2}} \int_{E_{i 0}}^{\infty} \frac{\exp \left(z-\frac{E_{F}}{k_{B}}\right)}{\left[1+\exp \left(z-\frac{E_{F}}{k_{B} T}\right)\right]^{2}} k_{i}^{3}(z) d z$,

Where $z=E_{i} / k_{B} T, E_{F}$ is the Fermi level energy, $E_{i}$ is the energy of the holes, $k_{i}$ is the wavevector and $E_{i 0}$ is the maximum of the valence band $\left(i=\mathrm{L}, \sum\right.$ for SnTe),${ }^{3,8,9}$ here the hole energy is positive in this notation.

After considering the 4-fold degeneracy for the L-point and 12-fold degeneracy for the $\sum$ point, the total holes concentration $p_{\text {tot }}$ in SnTe can be expressed as

$p_{\text {tot }}=\sum_{i} p_{i}=4 p_{L}+12 p_{\Sigma}$ 
The valence band at the L-point is described by a non-parabolic dispersion given by Kane's two-band $\boldsymbol{k} \cdot \boldsymbol{p}$ model, ${ }^{14}$

$E_{L}(k)=\frac{\hbar^{2} k^{2}}{2 m_{0}}-\frac{E_{g}}{2}+\sqrt{\left(\frac{E_{g}}{2}\right)^{2}+\left(\frac{E_{g} \hbar^{2} k^{2}}{2 m_{l h}^{*}}\right)}$,

Where $E_{g}=0.3 \mathrm{eV}$ is the band gap of SnTe and $m l h *$ is the effective mass of the holes at the L-point.

The second lower lying valence bands at the $\sum$-point is parabolic with the dispersion given by

$E_{\Sigma}(k)=\emptyset_{\Sigma}^{0}+\frac{\hbar^{2} k^{2}}{2 m_{h h}^{*}}$

Where $\emptyset_{\Sigma}^{0}=0.3 \mathrm{eV}$ is the energy of the valence band edge and $m_{h h} *$ is the effective mass of the holes at the $\sum$-point. Assuming $m_{l h}{ }^{*}=0.1 \mathrm{~m}_{0}$ and $m_{h h}{ }^{*}=0.6 \mathrm{~m}_{0}$, we can calculate the $p_{\text {tot }}$ as a function of Fermi level energy below the VBE at the L-point. 


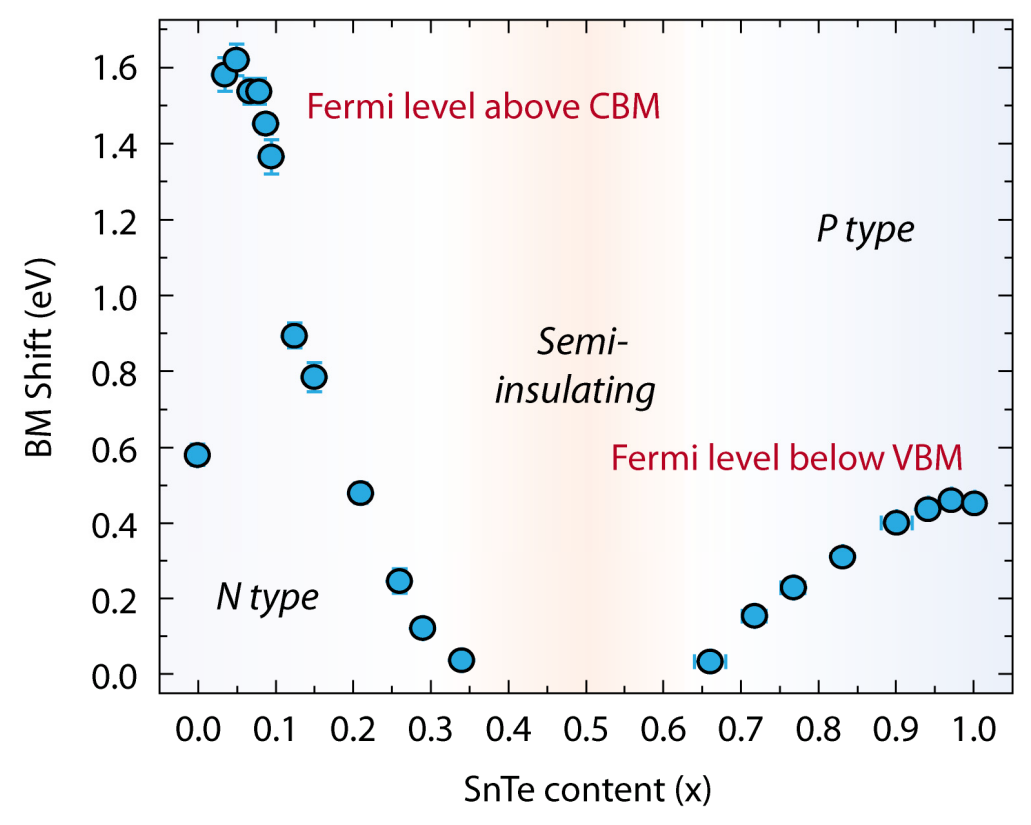

Figure S2. Burstein Moss shift in the $(\mathrm{SnTe})_{\mathrm{x}}(\mathrm{CdO})_{1-\mathrm{x}}$ nanocomposites. Burstein Moss $(\mathrm{BM})$ shift was calculated from the measured carrier concentration, as a function of SnTe content $\mathrm{x}$. The BM shift corresponds to Fermi level above CBE on the CdO-rich side and Fermi level below VBE on the SnTe-rich side, respectively.

The intrinsic energy gaps are then calculated correcting the absorption edge energy for the BM shift and including free charge carrier-induced renormalization effects including electronelectron and electron-ion interactions. ${ }^{8,9}$

For a n-type CdO-rich semiconductor,

$E_{g}=E_{A E}-E_{B M}(n)+E_{e-e}(n)+E_{e-i}(n)$,

For a p-type SnTe-rich semiconductor,

$E_{g}=E_{A E}-E_{B M}(p)+E_{h-h}(p)+E_{h-i}(p)$, 
Where $E_{A E}$ is the measured absorption edge, $E_{B M}(n / p)$ is the Burstein-Moss shift effect, $E_{e-e}(n)$ and $E_{e-i}(n)$ are the carrier-induced renormalizations due to the electron-electron correlation and electron-ion interactions, $E_{h-h}(p)$ and $E_{h-i}(p)$ are the carrier-induced renormalizations due to the hole-hole correlation and hole-ion interactions, respectively.

The intrinsic bandgap $E_{g}$ of the composite represented by the red circles in Fig. 1c., clearly shows a non-monotonic composition dependence with the maximum gap of $\sim 2.7 \mathrm{eV}$ for $\mathrm{x}=0.35$.

This unusual, positive bowing of the direct band gap can be understood by interpolation of the $\Gamma$ and $\mathrm{L}$ point critical point energies between $\mathrm{CdO}$ and $\mathrm{SnTe}$. The composition dependence of the intrinsic band gap can be expressed in terms of the bowing equation,

For the direct band gap in $\Gamma$ point,

$E_{g \Gamma}^{S n T e C d O}(x)=E_{g \Gamma}^{S n T e} x+E_{g \Gamma}^{C d O}(1-x)-b_{\Gamma} x(1-x)$

For the direct band gap in L point,

$E_{g L}^{S n T e C d O}(x)=E_{g L}^{S n T e} x+E_{g L}^{C d O}(1-x)-b_{L} x(1-x)$

Where $E_{g \Gamma}^{S n T e C d O}, E_{g \Gamma}^{S n T e}$ and $E_{g \Gamma}^{C d O}$ are direct band gap of $(\mathrm{SnTe})_{\mathrm{x}}(\mathrm{CdO})_{1-\mathrm{x}}, \mathrm{SnTe}$ and $\mathrm{CdO}$ in $\Gamma$ point, respectively. $E_{g L}^{S n T e C d O}, E_{g L}^{S n T e}$ and $E_{g L}^{C d O}$ are direct band gap of $(\mathrm{SnTe})_{\mathrm{x}}(\mathrm{CdO})_{1-\mathrm{x}}, \mathrm{SnTe}$ and $\mathrm{CdO}$ in L point, respectively. $\mathrm{x}$ is SnTe content. Fitting of the intrinsic band gap yields a bowing parameters: $b_{\Gamma}=1.75 \mathrm{eV}$ and $b_{L}=2.85 \mathrm{eV}$. 


\section{Microstructure analysis}

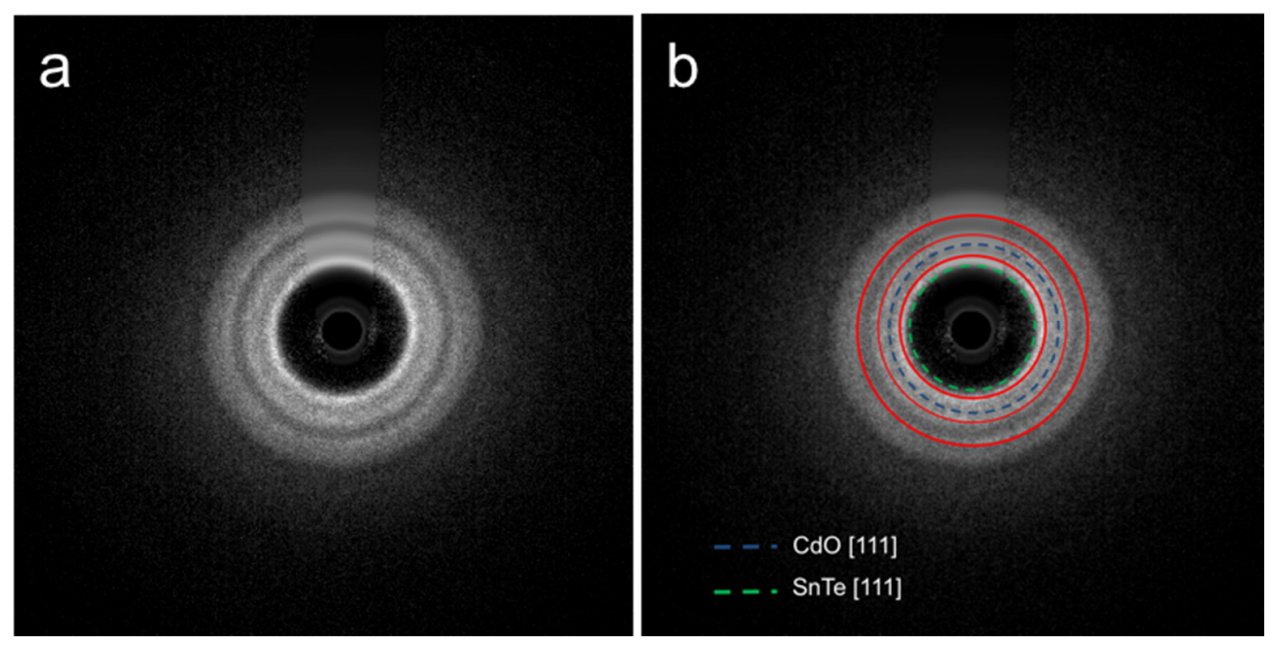

Figure S3. SAD pattern for $45 \%$ SnTe sample. (a) Whole patterns after background subtraction used to measured d-spacings. (b) Same pattern overlaid with $\{111\}$ diffraction rings for standard $\mathrm{CdO}$ and $\mathrm{SnTe}$, respectively, blue and green dotted line. Red rings represent the radial distance from where d-spacings were extracted.

Fig. S3 presents a SAD pattern - after background subtraction, beam stop cropping and contrast enhancement, which is overlaid with red rings in the Fig. S3b indicating the 3 first set of diffractions. These are corresponding to $\{111\},\{002\}$ and $\{022\}$ diffraction families. Comparison with theoretical $\mathrm{CdO}$ and SnTe lattice parameters for $\{111\}$ planes is indicated, respectively, by the blue ring and green ring. The mean lattice parameter for the sample with $45 \% \mathrm{SnTe}$ was determined as $5.265 \AA( \pm 0.05 \AA)$. For comparison, we also present full SAD patterns from $10 \%$ SnTe and 73\% SnTe, respectively, in Figs. S4a and S4b.

Table S1 summarizes d-spacing values extracted for all samples studied and described in the manuscript. Diffraction planes with an index higher than (022) were not precise for measurement of the sample with $45 \%$ SnTe. 


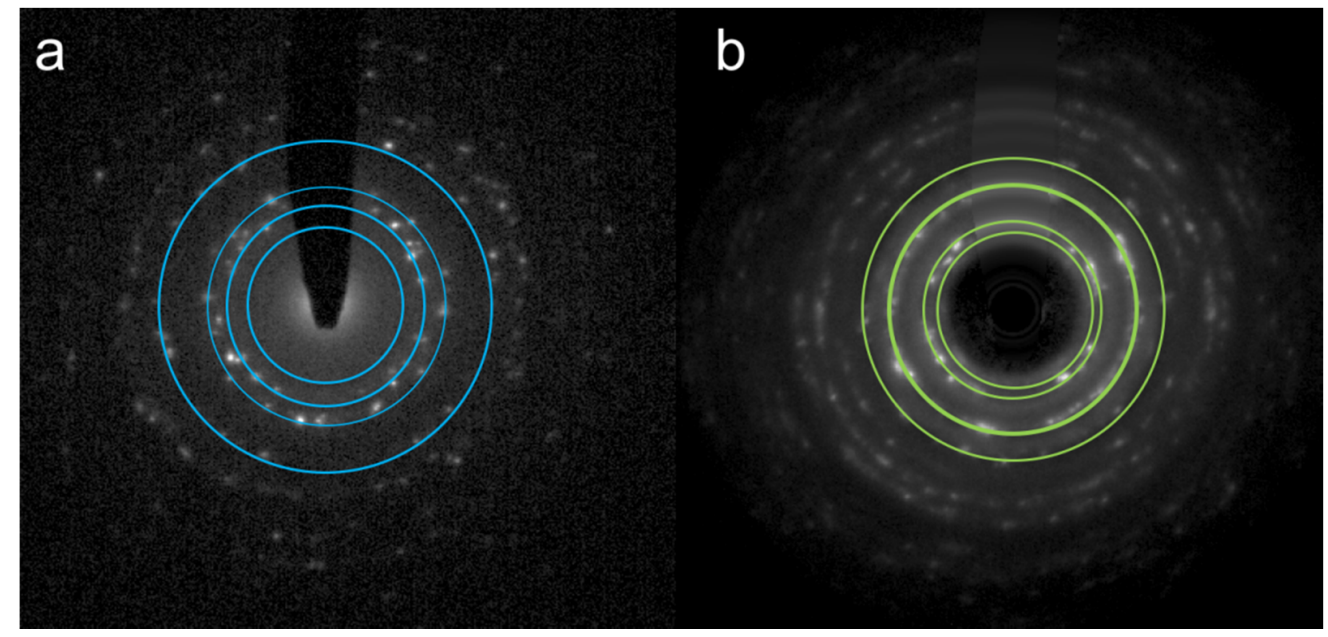

Figure S4. SAD patterns of $10 \% \mathrm{SnTe}$ (a) and $73 \% \mathrm{SnTe}$ (b) overlaid with the measured ring positions.

\begin{tabular}{|c|c|c|c|c|}
\hline $\begin{array}{c}\text { d-spacing } \\
\text { CdO (nm) }\end{array}$ & $\begin{array}{c}\text { d-spacing } \\
\mathbf{1 0 \%} \text { SnTe (nm) }\end{array}$ & $\begin{array}{c}\text { d-spacing } \\
45 \% \text { SnTe }(\mathrm{nm})\end{array}$ & $\begin{array}{c}\text { d-spacing } \\
\mathbf{7 3 \%} \text { SnTe (nm) }\end{array}$ & $\begin{array}{c}\text { d-spacing } \\
\text { SnTe (nm) }\end{array}$ \\
\hline$[111] 0.271$ & $\mathbf{0 . 2 8 7}$ & 0.304 & $\mathbf{0 . 3 6 5}$ & {$[111] 0.366$} \\
\hline$[002] 0.235$ & $\mathbf{0 . 2 4 9}$ & 0.263 & $\mathbf{0 . 3 1 6}$ & {$[002] 0.317$} \\
\hline$[022] 0.166$ & $\mathbf{0 . 1 7 6}$ & 0.189 & $\mathbf{0 . 2 2 6}$ & {$[022] 0.227$} \\
\hline$[113] 0.136$ & $\mathbf{0 . 1 5 0}$ & - & $\mathbf{0 . 1 8 9}$ & {$[113] 0.191$} \\
\hline
\end{tabular}

Table S1. Comparison of measured d-spacings for 10\%, 45\% and 73\% SnTe samples with standard $\mathrm{CdO}$ and SnTe. 


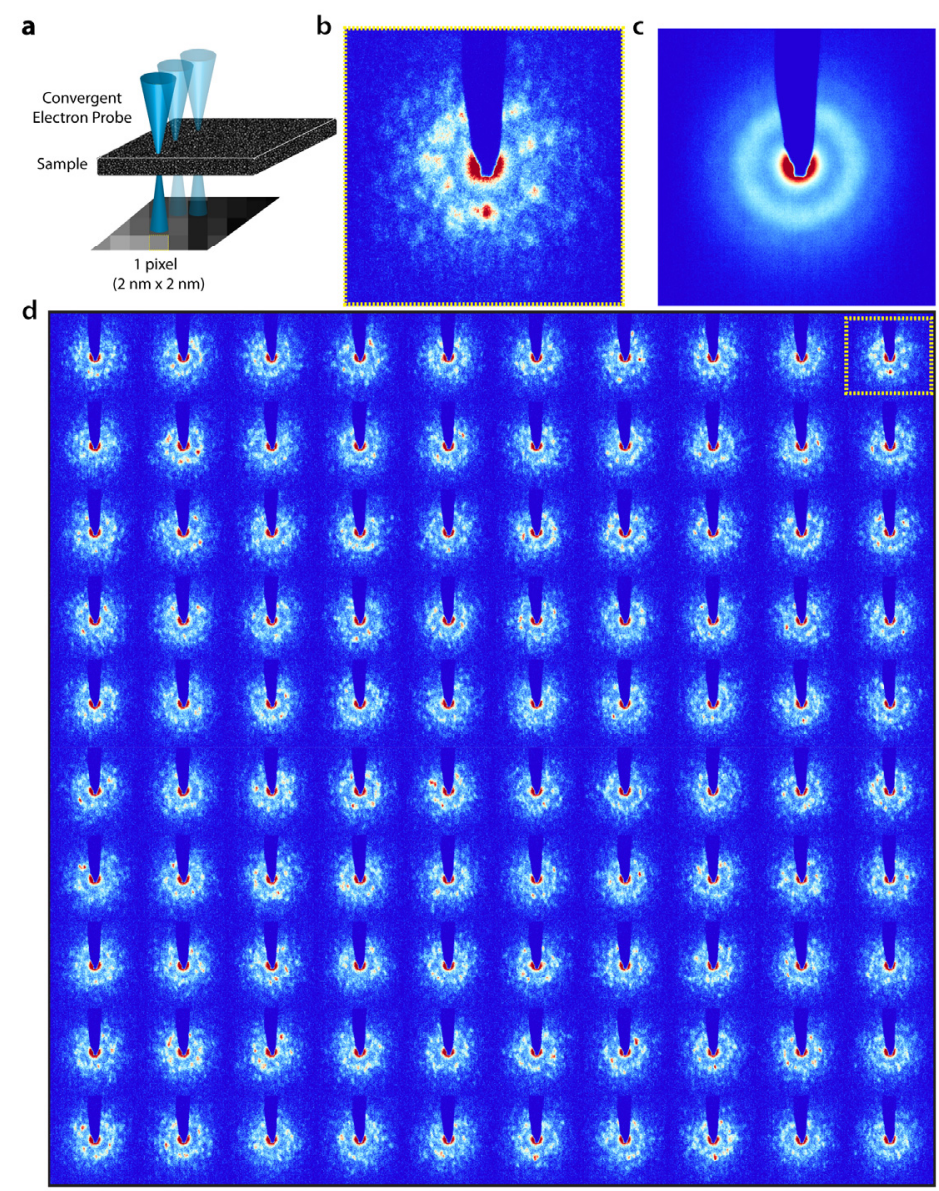

Figure S5. Nanobeam diffraction for semi-insulating $(\mathrm{SnTe})_{\mathrm{x}}(\mathrm{CdO})_{1-\mathrm{x}}$ nanocomposite with $\mathrm{x}=0.45$. (a) Schematic of nanobeam diffraction collection under STEM mode, each pattern is collected from a $2 \mathrm{~nm}$ by $2 \mathrm{~nm}$ area. (b) A single pattern extracted from the full series demonstrating presence of crystallites within the film. (c) An average of the full series of 100 patterns shown in d.

Fig. S5 presents nanobeam diffraction patterns collected as a series of 10 by 10 pixels with a step of $2 \mathrm{~nm}$ covering an area of $20 \mathrm{~nm}$ by $20 \mathrm{~nm}$ within the film (as sketched in Fig. S5a). ${ }^{15}$ A beam of $1.8 \mathrm{~nm}$ of diameter was used with a convergence angle of $0.96 \mathrm{mrad}$ under STEM mode. We can observe a speckled pattern for each of the nanodiffraction images as shown in Fig. S5b, corresponding to a presence of small crystallites. Averaging of the 100 patterns (shown in Fig. S5d) results in a diffuse pattern (Fig. S5c), similar to that observed in SAD and XRD (Fig. 2f and 
3a). This demonstrates that the intermediate composition film is a composite of small misoriented crystallites.

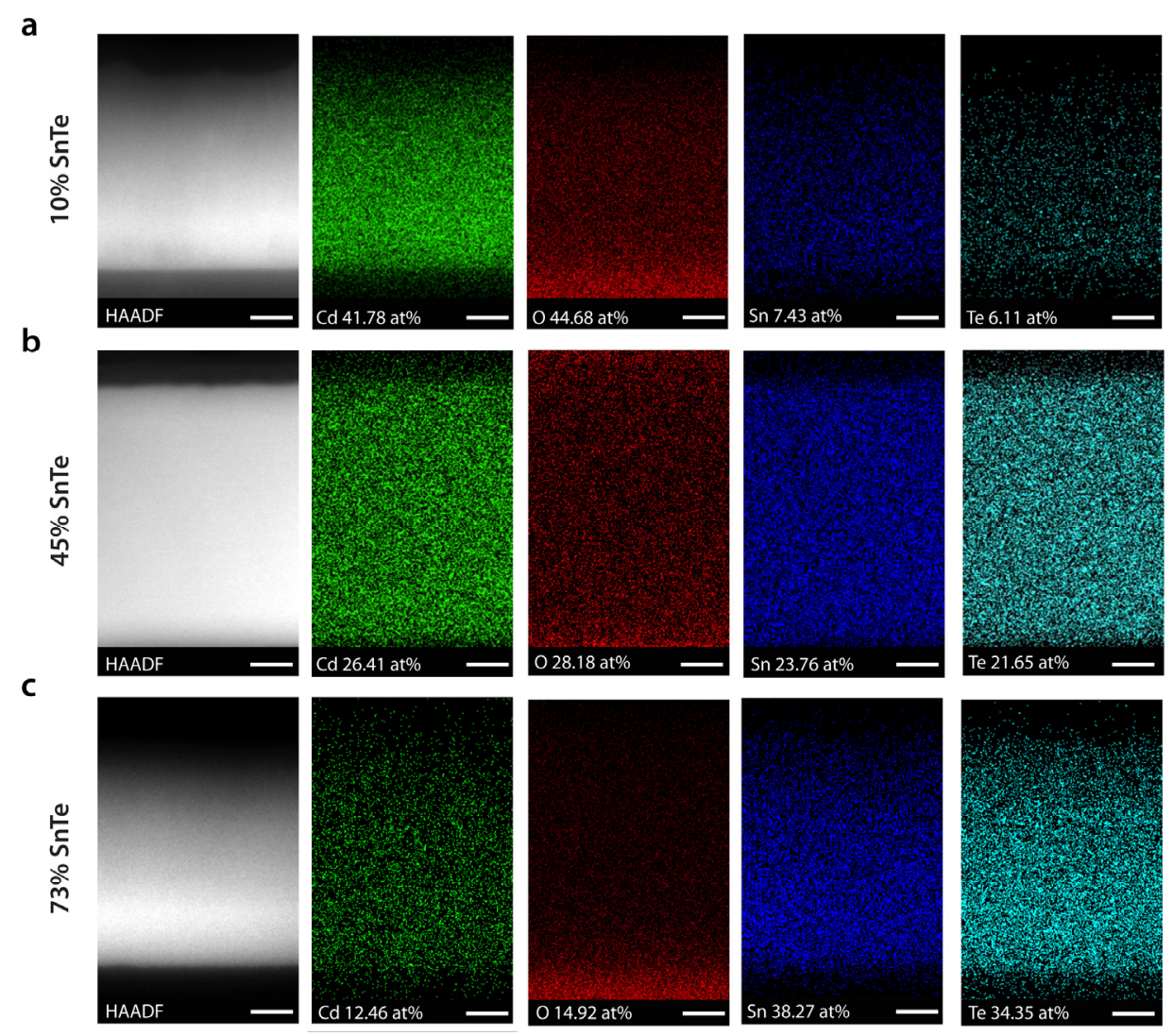

Figure S6. 2D EDX element mapping. 2D EDX element mapping for the detected 4 elements, Cd, $\mathrm{O}$, Sn, and Te, respectively, for $(\mathrm{SnTe})_{\mathrm{x}}(\mathrm{CdO})_{1-\mathrm{x}}$ nanocomposites with $10 \%$ (a), 45\% (b) and 73\% (c) of SnTe (top to bottom rows), alongside their respective HAADF spectrum image shown on the first column. The averaged elemental composition is shown at the bottom of each map. Scale bar for all micrographs is $50 \mathrm{~nm}$.

Fig. S6 shows a montage of two-dimensional energy dispersive spectroscopy (EDX) elemental mapping of representative samples for $\mathrm{Cd}, \mathrm{O}, \mathrm{Sn}$ and Te alongside their corresponding High angle annular dark filed (HAADF) spectrum image. Quantification process of spectra using 
L $\alpha$-lines was performed using a Cliff-Lorimer approach within the Bruker ESPRIT ${ }^{\circledR}$ software. This method is based on the intensities of measured peaks of L $\alpha$-lines relative to calculated or measured intensities of standards. ${ }^{16}$ The averaged element concentration is indicated for each of the maps. The calculated $\mathrm{Cd} /(\mathrm{Sn}+\mathrm{Te})$ atomic ratios of $3.085,0.581$ and 0.171 , respectively, for the samples with $10 \%$ of SnTe, $45 \%$ of SnTe and $73 \%$ of SnTe, are in agreement with those values obtained by RBS. In addition, these films don't exhibit any apparent element separation or aggregation, demonstrating that all elements are very homogeneously distributed across the layer at this resolution of $2 \mathrm{~nm}$ range.

\section{Composition of $(\mathrm{SnTe})_{\mathrm{x}}(\mathrm{CdO})_{1-\mathrm{x}}$ composites.}

a

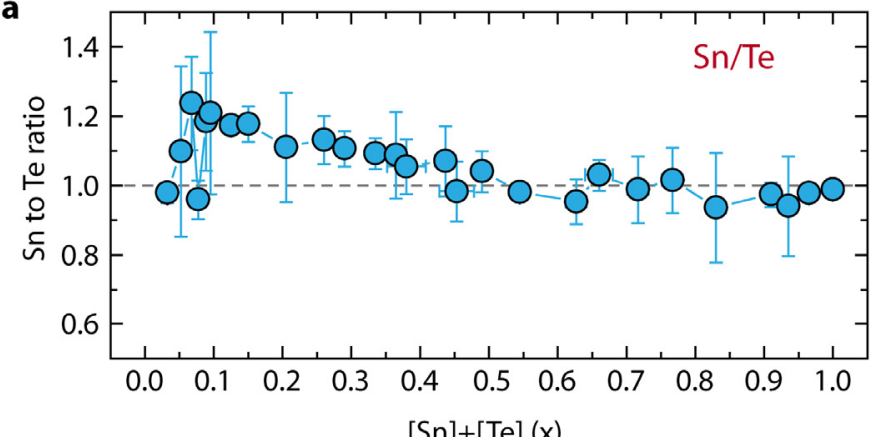

b

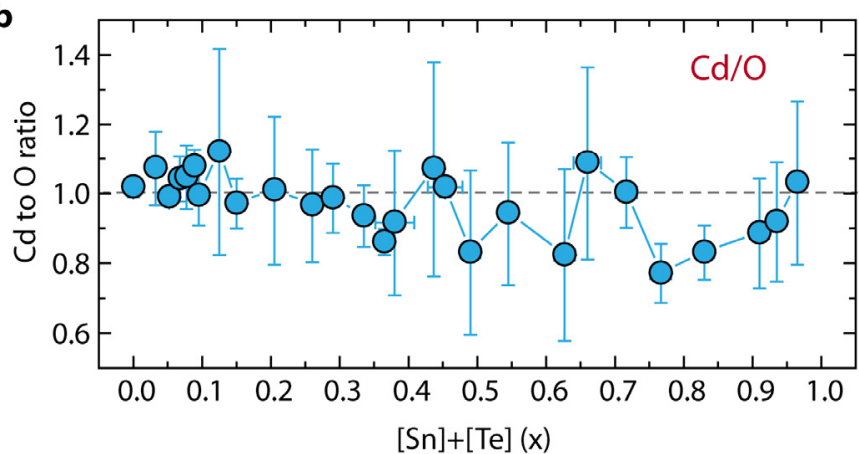

Figure S7. Composition of $(\mathrm{SnTe})_{\mathrm{x}}(\mathrm{CdO})_{1-\mathrm{x}}$ nanocomposites. (a) $\mathrm{Sn}$ to Te ratio and (b) $\mathrm{Cd}$ to $\mathrm{O}$ ratio dependence on the nominal SnTe content $\mathrm{x}$. RBS results indicating the $\mathrm{Sn}$ to Te ratio and $\mathrm{Cd}$ to $\mathrm{O}$ ratio remain almost constant over the entire composition range. 
The composition of the $(\mathrm{SnTe})_{x}(\mathrm{CdO})_{1-\mathrm{x}}$ nanocomposites measured using Rutherford backscattering spectroscopy (RBS), is shown in Fig. S7. The details of the experimental procedures are given in the Method Section. The $\mathrm{Sn}$ to Te ratio and $\mathrm{Cd}$ to $\mathrm{O}$ ratio were calculated by fitting the RBS curve using SIMNRA software. RBS results indicate that $\mathrm{Sn}$ to Te ratio and Cd to $\mathrm{O}$ ratio remain almost constant over the most of the composition range, although a $\mathrm{Sn}$ excess is observed in the CdO-rich region and a slight $\mathrm{O}$ excess is observed in the SnTe-rich region. The lower sensitivity of RBS to light elements, such as O leads to large error bars in Fig. S7. These results suggest that the deposition mostly preserves stoichiometry of the targets and the composition of the synthesized material can be represented by the SnTe mole fraction $\mathrm{x}$ in the composite $(\mathrm{SnTe})_{\mathrm{x}}(\mathrm{CdO})_{1-\mathrm{x} .}$

\section{Synchrotron X-Ray Diffraction}
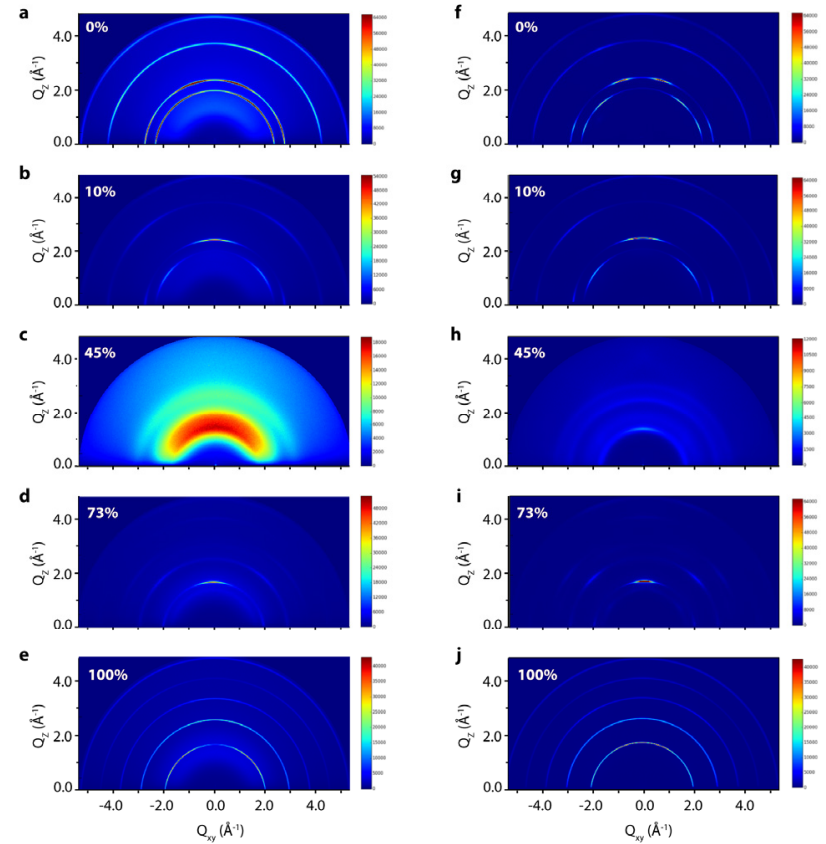

Figure S8. Two-dimensional XRD patterns. Two-dimensional XRD maps of $(\mathrm{SnTe})_{\mathrm{x}}(\mathrm{CdO})_{1-\mathrm{x}}$ nanocomposites grown on glass (a-e) and $\mathrm{Si}(\mathrm{f}-\mathrm{j})$ substrates over the entire composition range, respectively. 
Data shown in Fig. 3 were obtained by integrating 2D images shown in Fig. S8. Spectra were integrated between $5^{\circ}<\chi<175^{\circ}$ (Chi is the polar angle) using the WxDiff software package. ${ }^{17}$ The grazing incidence angle was $2^{\circ}$. On the glass substrate the patterns can be matched to $\mathrm{CdO}$ for $\mathrm{x}<0.13$ and SnTe $\mathrm{x}>0.73$. Broad diffusion halo was seen in the nanocomposite of $45 \% \mathrm{SnTe}$ grown on glass. After reducing the strong signal related to glass, the nanocomposite on Si with similar composition show broad diffusion ring which is consistent with, discussed above, a nanocrystalline nature of the film structure.

a

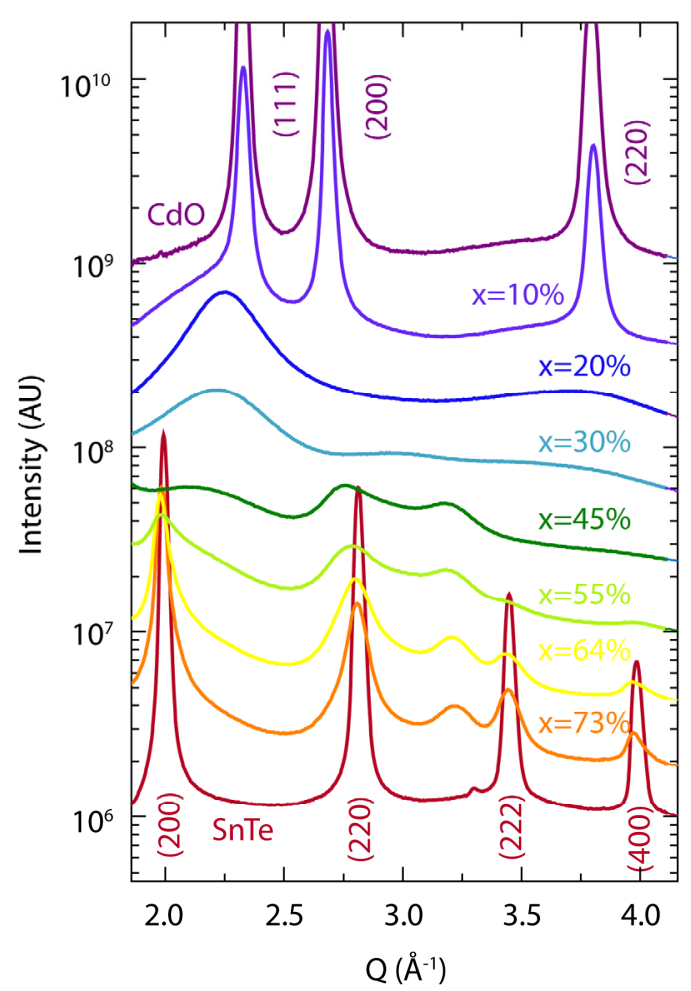

b

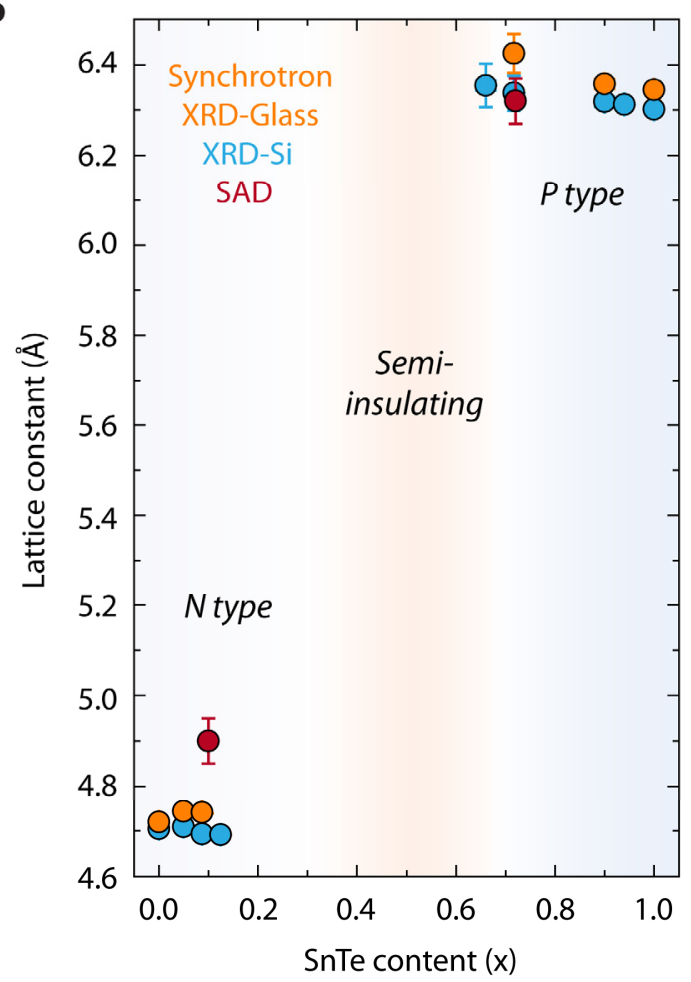

Figure S9. Crystal structure of $(\mathrm{SnTe})_{\mathrm{x}}(\mathrm{CdO})_{1-\mathrm{x}}$ nanocomposites. (a) XRD patterns for $(\mathrm{SnTe})_{\mathrm{x}}(\mathrm{CdO})_{1-\mathrm{x}}$ grown on $\mathrm{Si}$ substrate over the entire composition range. (b) Calculated lattice constants obtained from Synchrotron XRD and SAD as functions of SnTe content $\mathrm{x}$. The error bars are estimated by considering different samples and diffraction peaks width. The error bars for the SnTe content are as large as symbol size. 
The crystal structural analysis was performed by 2D wide angle X-ray diffraction. The results are shown in Fig. 3. Broad and diffuse peaks were seen in the intermediate composition range, indicating a nanocrystalline in the material. To determine if the peaks in this region are due to the amorphous substrate or the film itself, a set of samples were grown on single crystal Si substrates to reduce any signal related to the substrate. The data shown in Fig. S9a were obtained by integrating 2D images shown in Fig. S8. For the samples grown the single crystal Si substrates the SnTe matching pattern can be seen for $\mathrm{x}>0.55$. Also, an observation of diffuse peaks in this intermediate composition region is consistent with the nano-crystalline film. There is no obvious shift in the lattice parameter in each majority phase region.

\section{Schematics of the charge transfer induced formation of $(\mathrm{SnTe})_{\mathrm{x}}(\mathrm{CdO})_{1-\mathrm{x}}$ nanocomposites.}

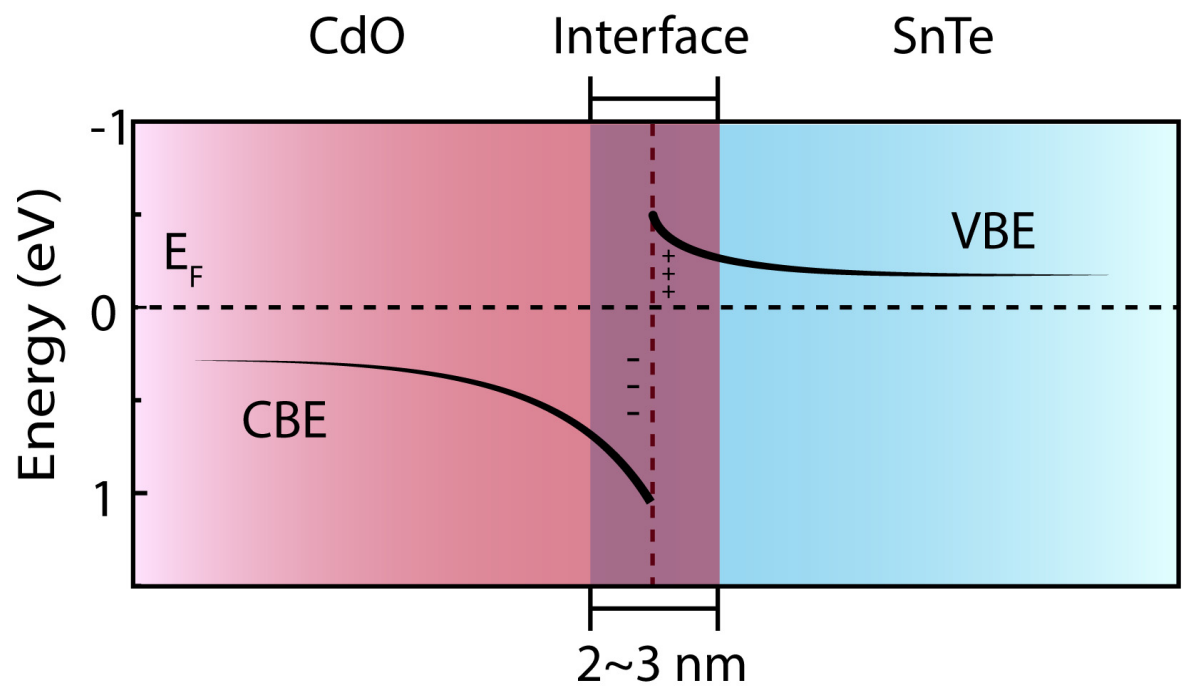

Figure S10. Band bending at the $\mathrm{CdO} / \mathrm{SnTe}$ interface. Schematics of the charge transfer induced band bending at the interface of $\mathrm{CdO} / \mathrm{SnTe}$. The width of the electron and hole accumulation interface region is limited to 2 to $3 \mathrm{~nm}$. The charge transfer reduces the interface energy and stabilizes $\mathrm{CdO}$ and/or SnTe nanocrystals with the size comparable to the interface width. 
Fig. S10 shows how the large type III band offset between $\mathrm{CdO}$ and SnTe results in a charge transfer and the band bending at the interface of those two materials. There are no depletion regions or barriers for charge transfer at the $\mathrm{CdO} / \mathrm{SnTe}$ interface. The charge accumulation region with 2 to $3 \mathrm{~nm}$ width is formed at the interface.

a

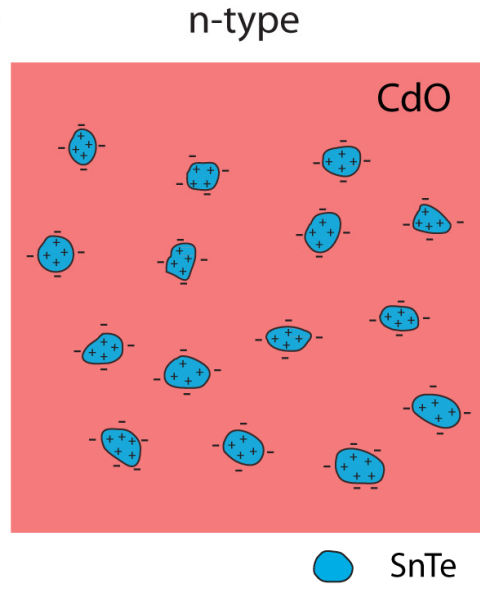

b



C

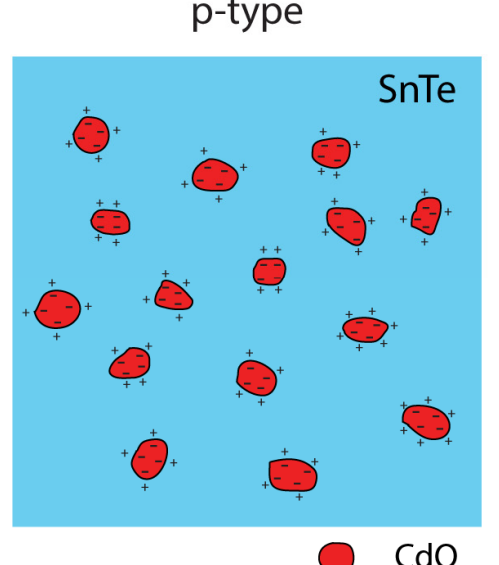

Figure S1 1. Schematic formation of $(\mathrm{SnTe})_{x}(\mathrm{CdO})_{1-x}$ nanocomposites. (a) n-type CdO-rich region, (b) Semi-insulating region, (c) p-type SnTe-rich region.

As the charge transfer reduces the interface energy the CdO-SnTe system will tend to maximize the total interface volume. Thus, as is schematically shown in Fig. S11a (Fig. S11c) in the cases of CdO-rich (SnTe-rich) material small, isolated crystallites of the minority phase SnTe (CdO) will be formed. The size of the crystallites is directly related to the width of the interface region. For the intermediate compositions the material forms a network of electrostatically bound nanocrystallites of $\mathrm{CdO}$ and SnTe shown in Fig. S1 1b. 


\section{REFERENCES:}

(1) Burbano, M.; Scanlon, D. O.; Watson, G. W. J. Am. Chem. Soc. 2011, 133, 15065-15072.

(2) Littlewood, P. B.; Mihaila, B.; Schulze, R. K.; Safarik, D. J.; Gubernatis, J. E.; Bostwick, A.; Rotenberg, E.; Opeil, C. P.; Durakiewicz, T.; Smith, J. L.; Lashley, J. C. Phys. Rev. Lett. 2010, $105,086404$.

(3) Speaks, D. T.; Mayer, M. A.; Yu, K. M.; Mao, S. S.; Haller, E. E.; Walukiewicz, W. J. Appl. Phys. 2010, 107, 113706.

(4) Rabii, S. Phys. Rev. 1969, 182, 821-828.

(5) Tung, Y. W.; Cohen, M. L. Phys. Rev. 1969, 180, 823-826.

(6) Burstein, E. Phys. Rev. 1954, 93, 632-633.

(7) Moss, T. S.; Hawkins, T. D. F. Infrared Phys. 1961, 1, 111-115.

(8) Berggren, K. F.; Sernelius, B. E. Phys. Rev. B 1981, 24, 1971-1986.

(9) Walukiewicz, W. Phys. Rev. B 1990, 41, 10218-10220.

(10) Baer, W. S. Phys. Rev. 1967, 154, 785-789.

(11) Morkoc, H.; Ozgur, U., Zinc Oxide: Fundamentals, Materials and Device Technology; John Wiley and Sons, Weinheim, 2008.

(12) Rogers, L. J. Phys. D: Appl. Phys. 1968, 1, 845.

(13) Bernick, R.; Kleinman, L. Solid State Commun. 1970, 8, 569-575.

(14) Kane, E. O. J. Phys. Chem. Solids 1957, 1, 13.

(15) Gammer, C.; Ozdol, V. B.; Liebscher, C. H.; Minor, A. M. Ultramicroscopy 2015, 155, 110.

(16) Cliff, G.; W., L. G. J. Microsc. 1975, 103, 203-207.

(17) Mannsfeld, S. C.; Tang, M. L.; Bao, Z. Adv. Mater. 2011, 23, 127-131. 